\title{
GW23-e0170 INTERLEUKIN-6-MEDIATED UPREGULATION OF BRAIN NATRIURETIC PEPTIDE EXPRESSION VIA THE JAK2/ STAT3 SIGNALLING PATHWAY IN NEONATAL RAT CARDIOMYOCYTES
}

doi:10.1136/heartjnl-2012-302920d.28

${ }^{1}$ Wen-Jing Xia, ${ }^{2} Y_{i}-Y_{i}$ Huang, ${ }^{1}$ Jian-Gui He. ${ }^{1}$ Department of Cardiology, First Affiliated Hospital of Sun Yat-sen University, Guangzhou, China; ${ }^{2}$ Department of Emergency, First Affiliated Hospital of Sun Yat-sen University, Guangzhou, China

Objectives We have shown that interleukin-6 (IL-6) upregulates brain natriuretic peptide (BNP) expression in rat cardiomyocytes under ischaemic conditions, partly via the transforming growth factor $\beta 1$ (TGF- $\beta 1$ )/SMAD2 signal pathway. Studies have shown that the IL-6/Janus kinase 2 (JAK2)/signal transducer and activator of transcription 3 (STAT3) signalling pathway plays a significant role in myocardial ischaemic injury. We have questioned whether JAK2 and STAT3 proteins also participate in IL-6-mediated regulation of BNP in neonatal rat cardiomyocytes.

Methods Direct drug intervention with IL- 6 showed that IL- 6 independently activates the JAK2/STAT3 pathway in neonatal rat cardiomyocytes.

Results Moreover, increased phosphorylation of the STAT3 protein was first detectable after 5 min of IL- 6 stimulation; phosphorylation was highest at $15 \mathrm{~min}$ and returned to basal level within $1 \mathrm{~h}$. A specific inhibitor was then chosen to investigate the role of the JAK2/STAT3 pathway. Pretreatment with $10 \mathrm{mM}$ AG490, a specific inhibitor for JAK2 protein, could suppress the increase in BNP levels induced by the administration of $10 \mathrm{ng} / \mathrm{ml}$ of IL- 6 , and maximal inhibition is achieved at a $50 \mathrm{mM}$ concentration of AG490. Furthermore, combinational inhibition with neutralising antibody, which blocks the TGF- $31 /$ SMAD2 pathway, and AG490 failed to completely suppress the increase in BNP levels.

Conclusions Thus, apart from the TGF- $\beta 1 / \mathrm{SMAD} 2$ signalling pathway, the JAK2/STAT3 pathway is also important in the IL-6mediated regulation of BNP in neonatal cardiomyocytes; other signalling pathways may also be involved in this process. 